\title{
Continuity Equation in Presence of a Non-Local Potential in Non-Commutative Phase-Space
}

\author{
Ilyas Haouam \\ Laboratoire de Physique Mathématique et de Physique Subatomique (LPMPS), Université Frères Mentouri, Constantine, Algeria \\ Email: ilyashaouam@live.fr
}

How to cite this paper: Haouam, I. (2019) Continuity Equation in Presence of a Non-Local Potential in Non-Commutative Phase-Space. Open Journal of Microphysics, 9, 15-28.

https://doi.org/10.4236/ojm.2019.93003

Received: January 18, 2019

Accepted: August 26, 2019

Published: August 29, 2019

Copyright ( 2019 by author(s) and Scientific Research Publishing Inc. This work is licensed under the Creative Commons Attribution International License (CC BY 4.0).

http://creativecommons.org/licenses/by/4.0/

(c) (i) Open Access

\begin{abstract}
We studied the continuity equation in presence of a local potential, and a non-local potential arising from electron-electron interaction in both commutative and non-commutative phase-space. Furthermore, we examined the influence of the phase-space non-commutativity on both the locality and the non-locality, where the definition of current density in commutative phase-space cannot satisfy the condition of current conservation, but with the steady state, in order to solve this problem, we give a new definition of the current density including the contribution due to the non-local potential. We showed that the calculated current based on the new definition of current density maintains the current. As well for the case when the noncommutativity in phase-space considered, we found that the conservation of the current density completely violated; and the non-commutativity is not suitable for describing the current density in presence of non-local and local potentials. Nevertheless, under some conditions, we modified the current density to solve this problem. Subsequently, as an application we studied the Frahn-Lemmer non-local potential, taking into account that the employed methods concerning the phase-space non-commutativity are both of Bopp-shift linear transformation through the Heisenberg-like commutation relations, and the Moyal-Weyl product.
\end{abstract}

\section{Keywords}

Continuity Equation, Non-Local Potential, Non-Commutative Schrödinger Equation, Phase-Space Non-Commutativity, Frahn-Lemmer Potential, Moyal Product, Bopp-Shift Linear Transformation

\section{Introduction}

In physics sometimes the largest descriptive equations reach their limits. A considered number of physics equations are only approximations. What physicists 
really want is not an approximation; they want equations that connect the world's behaviors directly to the foundations of reality such as the motion equations, the continuity equations, etc. We can consider that the continuity equation is one of the equations that describe the basic concepts, where it expresses the kinematical aspect of a symmetry and is a useful auxiliary equation.

In the last years, in the development of nanotechnology, the transport properties of nanodevices more and more become important, and it is very interesting to understand how the current flows inside the nanodevices, and how the current density gives the information about the heat dissipation for example [1], as well as in the elementary particle physics, for the quarks and the gluons which have a color charge [2] [3] (in the theory of quantum chromodynamics (QCD)), in which it is always conserved such as the electric charge. There is a continuity equation for such a color charge current (given at the gluon field strength tensor).

There are numerous quantities which are often or always conserved, such as the baryon number which is proportional to the number of quarks minus the number of antiquarks, also the lepton number, the isospin (term used to describe groups of particles which have nearly the same mass, such as the proton and the neutron), etc. Which means, in order to investigate their own conservation laws, we have to reach their continuity equations. Where the continuity equation is an equation that describes the transport of some quantity, furthermore there must be a quantity $x$ that can flow or move (such as the energy, the particle charge...), with $\rho_{x}$ being the volume density of this quantity, the way this quantity flows is described by its flux denoted $J_{x}$. Knowing that, the continuity equation is another form of the conservation law, will be meaningful if it is applied to a conserved quantity, and can be expressed in the integral form, or in the differential form by the divergence theorem, it is related always to the conservation of probability in quantum mechanics (QM).

In this paper, we derive the continuity equation for a particle subjected to non-local and local potentials [4] in non-commutative phase-space (NCPS). Why do we care about the non-local potential and the non-commutative geometry (NCG)? The reason behind that, the Schrödinger equation in the presence of a non-local potential has been the subject of many investigations for several years [5] [6] [7] such as, in the calculations of the transport properties of the nanodevices through the density functional theory and the Green's function theory [8] [9] yonder, there are many cases where the non-local potential is present. In the scattering theories of nucleons and nuclei [10], the non-locality is generated by the exchange interaction between the nucleons in the nucleus (considered in Hartree-Fock type calculations) [11]. In addition, the nuclear optical potential describing the movement between colliding nuclei is also non-local [12] [13]. In most nuclear structure and reaction calculations yet, the non-locality has been assumed to be small, and several approximation methods have been introduced in the local potential model for reproducing its contributions.

This work is realized in the NCG due to the importance and the advantages of 
it in both quantum mechanics and quantum fields, and generally in physics today. Knowing that, the origin of the NCG is relative to the search for topological spaces ( $C^{*}$-algebras) of functions that are replaced by non-commutative algebras, later the NCG concept was rekindled by A. Connes and others [14] [15] [16] [17], who theorized the idea of a differential structure in a non-commutative framework, by studying and defining the cyclic cohomology. Where they showed that, the notion of differential calculations on varieties had a non-commutative equivalent. Then, this type of geometry found great support by many mathematical results: Characterizations of commutative von Neumann algebras, Gelfand-Naïmark theorem on $C^{\star}$-algebras, cyclic cohomology of $C^{\infty}(\mathrm{M})$ algebra, $\mathrm{K}$ theory of $C^{*}$-algebras, relations between Dirac operators and Riemannian metrics.

A non-commutative space theory replaces the non-commutativity of operators associated with space-time coordinates with a deformation in the algebra of functions defined on space-time, and the non-commutative version of the field theory is obtained by changing the commutative theory to the non-commutative one, this done by replacing ordinary fields with non-commutative fields and ordinary products with Moyal-Weyl products. To be more precise, N. Seiberg and E. Witten in the past few years made their famous article [18], which is from the most cited articles, encouraged a wide amount of interest in NCG, which became the mainstream for a couple of years.

It is worthwhile to mention that, the idea of NCPS is based essentially on the Seiberg-Witten map, the Bopp's shift method and the Moyal-Weyl product.

In this paper, our aim is not to solve the equations but to focus on extracting continuity equations. The plan of this paper is as follows: In Section 3, we derive the continuity equation for non-local and local potentials, taking as an application the Frahn-Lemmer non-local potential. In Section 4, we have the interesting result of our paper, which is represented in the non-commutative continuity equation for non-local and local potentials (with an application of the Frahn-Lemmer non-local potential).

\section{Review of the Non-Commutative Geometry}

The non-commutative geometry is the theory in which space may not commute anymore. Let us consider the operators of coordinates and momentum in a $\mathrm{d}$ dimensional non-commutative phase-space $x_{i}^{n c}$ and $p_{i}^{n c}$ respectively. Where the non-commutative phase-space operators satisfy the Heisenberg-like commutation relations [19]

$$
\left[x_{i}^{n c}, x_{j}^{n c}\right]=i \Theta_{i j},\left[p_{i}^{n c}, p_{j}^{n c}\right]=i \eta_{i j},\left[x_{i}^{n c}, p_{j}^{n c}\right]=i \hbar^{e f f} \delta_{i j}(i, j=1, \cdots, d),
$$

the effective Planck constant being

$$
\hbar^{e f f}=\hbar(1+\xi)
$$

where $\xi=\frac{\operatorname{Tr}(\Theta \eta)}{4 \hbar^{2}}$, the consistency condition is $\xi \ll 1$. With $\Theta_{i j}, \eta_{i j}$ are 
antisymmetric real constant $(d \times d)$ matrices and $\delta_{i j}$ is the identity matrix. Theoretical predictions for non-commutative systems (concerning the non-commutative parameters) have been compared to experimental data (the maximum absolute energy shifts allowed by the experiment), leading to bounds on the noncommutative parameters [19] [20]:

$$
\Theta \approx 4 \times 10^{-40} \mathrm{~m}^{2}, \eta \approx 1.76 \times 10^{-61} \mathrm{~kg}^{2} \cdot \mathrm{m}^{2} \cdot \mathrm{s}^{-2} .
$$

These above bounds will be suppressed due to the weak magnetic field used in the experiments $B \approx 5 \mathrm{mg}$.

Since the system in which we study the effects of non-commutativity, is three dimensional, we limit our calculations to the following non-commutative algebra

$$
\left[x_{i}^{n c}, x_{j}^{n c}\right]=i \epsilon_{i j k} \Theta_{k},\left[p_{i}^{n c}, p_{j}^{n c}\right]=i \epsilon_{i j k} \eta_{k},\left[x_{i}^{n c}, p_{j}^{n c}\right]=i \hbar^{e f f} \delta_{i j} \quad(i, j=1,2,3),
$$

take into account that we neglect the uncertainty relation between $x_{i}^{\text {nc }}$ and $p_{i}^{n c}$. With $\Theta_{i j}, \eta_{i j}$ are $(3 \times 3)$ antisymmetric matrices, and $\epsilon_{i j k}$ is Levi-Civita symbol and the summation convention is used. We have $\epsilon_{123}=\epsilon_{231}=\epsilon_{321}=-\epsilon_{321}=-\epsilon_{132}=-\epsilon_{231}=1$. If $i=j, j=k, \epsilon_{i j k}=0$, and $\Theta_{k}$, $\eta_{k}$ are the non-commutativity parameters. They are real-valued and antisymmetric constant matrices with the dimension of $(\text { lenght })^{2}$ and (momentum) $)^{2}$, respectively.

In the three dimensional commutative phase-space, the coordinates $x_{i}$ and momentum $p_{i}$ satisfy the usual canonical commutation relations

$$
\left[x_{i}, x_{j}\right]=\left[p_{i}, p_{j}\right]=0,\left[x_{i}, p_{j}\right]=i \hbar \delta_{i j}(i, j=1,2,3) \text {. }
$$

The non-commutative geometry Equation (1) is described at the level of fields and actions by the Moyal-Weyl product ( $\star$-product) [21] [22] [23]. Let $f$ and $g$ be two arbitrary functions from $\mathcal{R}^{D}$, we define $\star$-product as follows

$$
\begin{aligned}
& (f \star g)(x)=\exp \left[\frac{i}{2} \Theta_{a b} \partial_{x_{a}} \partial_{x_{b}}\right] f\left(x_{a}\right) g\left(x_{b}\right) \\
& =f(x) g(x)+\sum_{n=1}\left(\frac{1}{n !}\right)\left(\frac{i}{2}\right)^{n} \Theta^{a_{1} b_{1}} \cdots \Theta^{a_{n} b_{n}} \partial_{a_{1}} \cdots \partial_{a_{k}} f(x) \partial_{b_{1}} \cdots \partial_{b_{k}} g(x) .
\end{aligned}
$$

Note that in our calculations, we use the following $\star$-product properties:

The Complex conjugation

$$
(f(x) \star g(x))^{*}=g^{*}(x) \star f^{*}(x) .
$$

The $\star$-product under the integral sign

$$
\int(f \star g)(x) \mathrm{d}^{4} x=\int(g \star f)(x) \mathrm{d}^{4} x=\int(f g)(x) \mathrm{d}^{4} x .
$$

The non-commutative field theories for the low energies $\left(\Theta E^{2}<1\right)$ or the slowly varying fields effectively reduce to their commutative version due to the nature of the $\star$-product.

The non-commutative phase-space operators are related to the commutative phase-space one through the commutative Heisenberg-Weyl algebra in terms of the known Bopp-shift linear transformation which was introduced from the Equation (6) [24] [25], and it is given by 


$$
x_{i}^{n c}=x_{i}-\frac{1}{2 \hbar} \Theta_{i j} p_{j}, \quad p_{i}^{n c}=p_{i}+\frac{1}{2 \hbar} \eta_{i j} x_{j}
$$

If $\Theta=\eta=0$, the non-commutative phase-space framework will become commutative one.

\section{Schrödinger Equation in Presence of a Nonlocal Potential in Commutative Phase-Space}

In presence of a non-local potential $V_{N L}\left(\boldsymbol{r}, \boldsymbol{r}^{\prime}\right)$, the wave function obeys the following Schrödinger equation

$$
\frac{\boldsymbol{p}^{2}}{2 m} \psi(\boldsymbol{r}, t)+\int V_{N L}\left(\boldsymbol{r}, \boldsymbol{r}^{\prime}\right) \psi\left(\boldsymbol{r}^{\prime}, t\right) \mathrm{d} \boldsymbol{r}^{\prime}=i \hbar \frac{\partial}{\partial t} \psi(\boldsymbol{r}, t) .
$$

A non-local potential operating on a wave function [26] has the form

$$
\int V_{N L}\left(\boldsymbol{r}, \boldsymbol{r}^{\prime}\right) \psi\left(\boldsymbol{r}^{\prime}, t\right) \mathrm{d} \boldsymbol{r}^{\prime}=\int V_{N L}(\boldsymbol{r}, \boldsymbol{r}+\boldsymbol{s}) \psi(\boldsymbol{r}+\boldsymbol{s}, t) \mathrm{d} \boldsymbol{s},
$$

with $\boldsymbol{r}^{\prime}=\boldsymbol{r}+\boldsymbol{s}$, and $\mathrm{d} \boldsymbol{r}^{\prime}=\mathrm{d} \boldsymbol{s}$, taking into account that $\operatorname{Re}\left[V_{N L}\left(\boldsymbol{r}, \boldsymbol{r}^{\prime}\right)\right]=\operatorname{Re}\left[V_{N L}\left(\boldsymbol{r}^{\prime}, \boldsymbol{r}\right)\right]$ (symmetric), and using the Taylor series $\psi(\boldsymbol{r}+\boldsymbol{s})=\left(1+\boldsymbol{s} \frac{\partial}{\partial \boldsymbol{r}}+\frac{\boldsymbol{s}^{2}}{2} \frac{\partial^{2}}{\partial \boldsymbol{r}^{2}}+\frac{\boldsymbol{s}^{3}}{3 !} \frac{\partial^{3}}{\partial \boldsymbol{r}^{3}}+\cdots\right) \psi(\boldsymbol{r})=\mathrm{e}^{\frac{i s \boldsymbol{p}}{\hbar}} \psi(\boldsymbol{r})$. We find

$$
\int V_{N L}\left(\boldsymbol{r}, \boldsymbol{r}^{\prime}\right) \psi\left(\boldsymbol{r}^{\prime}, t\right) \mathrm{d} \boldsymbol{r}^{\prime}=\int V_{N L}(\boldsymbol{r}, \boldsymbol{r}+\boldsymbol{s}) \mathrm{e}^{\frac{i s p}{\hbar}} \mathrm{d} \boldsymbol{s} \psi(\boldsymbol{r}) .
$$

We can denote

$$
\begin{aligned}
& \int V_{N L}(\boldsymbol{r}, \boldsymbol{r}+\boldsymbol{s}) \mathrm{e}^{\frac{i s \boldsymbol{p}}{\hbar}} \mathrm{d} \boldsymbol{s} \psi(\boldsymbol{r}, t) \\
& =\int \mathrm{d} \boldsymbol{s} \mathrm{e}^{\frac{i s \boldsymbol{p}}{2 \hbar}} V_{N L}\left(\boldsymbol{r}-\frac{\boldsymbol{s}}{2}, \boldsymbol{r}+\frac{\boldsymbol{s}}{2}\right) \mathrm{e}^{\frac{i s \boldsymbol{p}}{2 \hbar}} \psi(\boldsymbol{r}, t)=V_{N L}(\boldsymbol{r}, \boldsymbol{p}) \psi(\boldsymbol{r}, t),
\end{aligned}
$$

then simply Equation (10) takes the form

$$
\frac{\boldsymbol{p}^{2}}{2 m} \psi(\boldsymbol{r}, t)+V_{N L}(\boldsymbol{r}, \boldsymbol{p}) \psi(\boldsymbol{r}, t)=i \hbar \frac{\partial}{\partial t} \psi(\boldsymbol{r}, t) .
$$

\subsection{Frahn-Lemmer Non-Local Potential}

In order to facilitate the calculations, and as an application concerning the non-local potential, we choose the Frahn-Lemmer potential [10] [27] defined as

$$
V_{N L}\left(\boldsymbol{r}, \boldsymbol{r}^{\prime}\right)=\mathcal{U}\left(\frac{1}{2}\left|\boldsymbol{r}+\boldsymbol{r}^{\prime}\right|\right) \mathcal{H}\left(\left|\boldsymbol{r}-\boldsymbol{r}^{\prime}\right|\right)
$$

where $\mathcal{U}, \mathcal{H}$ represent the local average value and the width of the non-locality respectively, knowing that, for simplicity we consider $\mathcal{U}\left(\frac{1}{2}\left|\boldsymbol{r}+\boldsymbol{r}^{\prime}\right|\right) \approx \mathcal{V}_{0}$ and $\mathcal{H}$ should be a normalized Gaussian function as

$$
\mathcal{H}\left(\left|\boldsymbol{r}-\boldsymbol{r}^{\prime}\right|\right)=\frac{1}{\left(\pi \beta^{2}\right)^{\frac{3}{2}}} \mathrm{e}^{-\frac{\left(\boldsymbol{r}-\boldsymbol{r}^{\prime}\right)^{2}}{\beta^{2}}},
$$


which is normalized so that

$$
\int \mathcal{H}\left(\left|\boldsymbol{r}-\boldsymbol{r}^{\prime}\right|\right) \mathrm{d} \boldsymbol{r}^{\prime}=1
$$

Then, our non-local potential takes the form [once the range parameter is very small, the non-local potential tends to $\approx \mathcal{V}_{0} \delta\left(\boldsymbol{r}-\boldsymbol{r}^{\prime}\right)$ ]

$$
V_{N L}\left(\boldsymbol{r}, \boldsymbol{r}^{\prime}\right)=\frac{\mathcal{V}_{0}}{\left(\pi \beta^{2}\right)^{\frac{3}{2}}} \mathrm{e}^{-\frac{\left(\left|\boldsymbol{r}-\boldsymbol{r}^{\prime}\right|\right)^{2}}{\beta^{2}}},
$$

with $\beta, \mathcal{V}_{0}$ are the non-locality range (typically takes on a value of $0.85 \mathrm{Fm}$ ) and the depth of the nuclear potential (Wood-Saxon function type) respectively.

The following equation, obtained by putting Equation (17) into Equation (10)

$$
\frac{\boldsymbol{p}^{2}}{2 m} \psi(\boldsymbol{r}, t)+\int \frac{\mathcal{V}_{0}}{\left(\pi \beta^{2}\right)^{\frac{3}{2}}} \mathrm{e}^{-\frac{\left(\boldsymbol{r}-\boldsymbol{r}^{\prime}\right)^{2}}{\beta^{2}}} \psi\left(\boldsymbol{r}^{\prime}, t\right) \mathrm{d} \boldsymbol{r}^{\prime}=i \hbar \frac{\partial}{\partial t} \psi(\boldsymbol{r}, t) .
$$

Using Equation (13), we have

$$
V_{N L}(\boldsymbol{r}, \boldsymbol{p})=\int \mathrm{d} \boldsymbol{s} \mathrm{e}^{\frac{i s p}{2 \hbar}} \frac{\mathcal{V}_{0}}{\left(\pi \beta^{2}\right)^{\frac{3}{2}}} \mathrm{e}^{-\frac{\left(\boldsymbol{r}-\frac{s}{2}-\boldsymbol{r}-\frac{s}{2}\right)^{2}}{\beta^{2}}} \mathrm{e}^{\frac{i s \boldsymbol{p}}{2 \hbar}}=\frac{\mathcal{V}_{0}}{\left(\pi \beta^{2}\right)^{\frac{3}{2}}} \mathrm{e}^{-\frac{(s)^{2}}{\beta^{2}+\frac{i}{\hbar} s p}},
$$

then, using the integral $\int_{+\infty}^{-\infty} \mathrm{e}^{-A^{2} \bar{s}^{2}+B s} \mathrm{~d} \boldsymbol{s}=\frac{\sqrt{\pi}}{A} \mathrm{e}^{\frac{B^{2}}{4 A^{2}}}$, with $A=\frac{1}{\beta}, \quad B=\frac{i}{\hbar} \boldsymbol{p}$ Equation (18) turns to

$$
\frac{\boldsymbol{p}^{2}}{2 m} \psi(\boldsymbol{r}, t)+\mathcal{V}_{0} \mathrm{e}^{-\frac{\boldsymbol{p}^{2} \beta^{2}}{4 \hbar^{2}}} \psi(\boldsymbol{r}, t)=i \hbar \frac{\partial}{\partial t} \psi(\boldsymbol{r}, t) .
$$

what we need here is to show the form of the Schrödinger equation in interaction with the Frahn-Lemmer non-local potential, knowing that if we want to solve the above equation, we have to use the Fourier transform to switch for the momentum representation ( $P$ representation), there the calculations shall be very easy, knowing that the equation will be time-independently considered, with $\boldsymbol{p}=\hbar \boldsymbol{k}$, Equation (20) becomes

$$
E-\frac{(\hbar \boldsymbol{k})^{2}}{2 m}=\mathcal{V}_{0} \mathrm{e}^{-\frac{k^{2} \beta^{2}}{4}}
$$

This explains the connection between nuclear potential and momentum. In another way, the strength of the potential decreases rapidly with increasing momentum.

\subsection{The Continuity Equation in Commutative Phase-Space}

The Schrödinger equation in presence of a non-local potential $V_{N L}\left(\boldsymbol{r}, \boldsymbol{r}^{\prime}\right)$ and a local potential $V_{L}(\boldsymbol{r})$ is written as follows

$$
-\frac{\hbar^{2}}{2 m} \nabla^{2} \psi(\boldsymbol{r}, t)+\int V_{N L}\left(\boldsymbol{r}, \boldsymbol{r}^{\prime}\right) \psi\left(\boldsymbol{r}^{\prime}, t\right) \mathrm{d} \boldsymbol{r}^{\prime}+V_{L}(\boldsymbol{r}) \psi(\boldsymbol{r}, t)=i \hbar \frac{\partial}{\partial t} \psi(\boldsymbol{r}, t) .
$$


The complex conjugate of the above equation written as

$$
-\frac{\hbar^{2}}{2 m} \nabla^{2} \psi^{\dagger}(\boldsymbol{r}, t)+\int V_{N L}^{*}\left(\boldsymbol{r}, \boldsymbol{r}^{\prime}\right) \psi^{\dagger}\left(\boldsymbol{r}^{\prime}, t\right) \mathrm{d} \boldsymbol{r}^{\prime}+V_{L}^{*}(\boldsymbol{r}) \psi^{\dagger}(\boldsymbol{r}, t)=-i \hbar \frac{\partial}{\partial t} \psi^{\dagger}(\boldsymbol{r}, t) .
$$

Here $*, \uparrow$ stand for the complex conjugation of the potentials and for the wave-functions successively.

In order to find the continuity equation, we have $\psi^{\dagger}(\boldsymbol{r}, t)$ Equation (23) and $\psi(\boldsymbol{r}, t)$ Equation (24), so that we obtain

$$
\begin{aligned}
& -\frac{\hbar^{2}}{2 m} \psi^{\dagger}(\boldsymbol{r}, t) \nabla^{2} \psi(\boldsymbol{r}, t)+\int \psi^{\dagger}(\boldsymbol{r}, t) V_{N L}\left(\boldsymbol{r}, \boldsymbol{r}^{\prime}\right) \psi\left(\boldsymbol{r}^{\prime}, t\right) \mathrm{d} \boldsymbol{r}^{\prime} \\
& +\psi^{\dagger}(\boldsymbol{r}, t) V_{L}(\boldsymbol{r}) \psi(\boldsymbol{r}, t)=i \hbar \psi^{\dagger}(\boldsymbol{r}, t) \frac{\partial}{\partial t} \psi(\boldsymbol{r}, t), \\
& -\frac{\hbar^{2}}{2 m} \psi(\boldsymbol{r}, t) \nabla^{2} \psi^{\dagger}(\boldsymbol{r}, t)+\int \psi(\boldsymbol{r}, t) V_{N L}^{*}\left(\boldsymbol{r}, \boldsymbol{r}^{\prime}\right) \psi^{\dagger}\left(\boldsymbol{r}^{\prime}, t\right) \mathrm{d} \boldsymbol{r}^{\prime} \\
& +\psi(\boldsymbol{r}, t) V_{L}^{*}(\boldsymbol{r}) \psi^{\dagger}(\boldsymbol{r}, t)=-i \hbar \psi(\boldsymbol{r}, t) \frac{\partial}{\partial t} \psi^{\dagger}(\boldsymbol{r}, t) .
\end{aligned}
$$

According to the subtraction of Equation (25) from Equation (26) we find

$$
\begin{aligned}
& -\frac{\hbar^{2}}{2 m} \nabla\left(\psi^{\dagger}(\boldsymbol{r}, t) \nabla \psi(\boldsymbol{r}, t)-\psi(\boldsymbol{r}, t) \nabla \psi^{\dagger}(\boldsymbol{r}, t)\right) \\
& +\int\left(\psi^{\dagger}(\boldsymbol{r}, t) V_{N L}\left(\boldsymbol{r}, \boldsymbol{r}^{\prime}\right) \psi\left(\boldsymbol{r}^{\prime}, t\right)-\psi(\boldsymbol{r}, t) V_{N L}^{*}\left(\boldsymbol{r}, \boldsymbol{r}^{\prime}\right) \psi^{\dagger}\left(\boldsymbol{r}^{\prime}, t\right)\right) \mathrm{d} \boldsymbol{r}^{\prime} \\
& +\psi^{\dagger}(\boldsymbol{r}, t) V_{L}(\boldsymbol{r}) \psi(\boldsymbol{r}, t)-\psi(\boldsymbol{r}, t) V_{L}^{*}(\boldsymbol{r}) \psi^{\dagger}(\boldsymbol{r}, t) \\
& =i \hbar \frac{\partial}{\partial t}\left(\psi^{\dagger}(\boldsymbol{r}, t) \psi(\boldsymbol{r}, t)\right) .
\end{aligned}
$$

If we multiply the above equation by the charge carried by the particle, we obtain the continuity equation of the corresponding particle, as in the case of the electron, we multiply by $(-e)$.

Equation (27) may be contracted as

$$
\frac{\partial \rho}{\partial t}+\nabla \boldsymbol{J}+\rho_{N L}+\rho_{L}=0
$$

The obtained continuity Equation (28) contains new quantities, which are the non-local current density $\rho_{N L}$, and the local density $\rho_{L}$, that is because of the consideration of the non-local and the local interactions in the Schrödinger equation, where

$$
\begin{aligned}
& \rho=J^{0}=\psi^{\dagger}(\boldsymbol{r}, t) \psi(\boldsymbol{r}, t)=|\psi(\boldsymbol{r}, t)|^{2} \\
& \boldsymbol{J}=\frac{-\hbar^{2}}{2 m}\left(\psi^{\dagger}(\boldsymbol{r}, t) \nabla \psi(\boldsymbol{r}, t)-\psi(\boldsymbol{r}, t) \nabla \psi^{\dagger}(\boldsymbol{r}, t)\right) \\
& \rho_{N L}=\int\left(\psi^{\dagger}(\boldsymbol{r}, t) V_{N L}\left(\boldsymbol{r}, \boldsymbol{r}^{\prime}\right) \psi\left(\boldsymbol{r}^{\prime}, t\right)-\psi(\boldsymbol{r}, t) V_{N L}^{*}\left(\boldsymbol{r}, \boldsymbol{r}^{\prime}\right) \psi^{\dagger}\left(\boldsymbol{r}^{\prime}, t\right)\right) \\
& \rho_{L}=\psi^{\dagger}(\boldsymbol{r}, t) V_{L}(\boldsymbol{r}) \psi(\boldsymbol{r}, t)-\psi(\boldsymbol{r}, t) V_{L}^{*}(\boldsymbol{r}) \psi^{\dagger}(\boldsymbol{r}, t)
\end{aligned}
$$

If the local potential $V_{L}(\boldsymbol{r})$ is Hermitian, implying that $\rho_{L}$ vanishes, which means that its symmetry maintained, these results are similar to the calculations of Changsheng Li and his colleagues [28].

In the steady state $\frac{\partial \rho}{\partial t}=0$, Equation (28) becomes 


$$
\nabla \boldsymbol{J}+\rho_{N L}=0 .
$$

If the non-local potential $V_{N L}\left(\boldsymbol{r}, \boldsymbol{r}^{\prime}\right)$ is a real diagonal matrix, the quantity $\rho_{N L}$ vanishes; its symmetry is maintained also. The current calculated from $\boldsymbol{J}$ is conserved since $\nabla \boldsymbol{J}=0$. However, in presence of a non-local potential, the quantity $\rho_{N L}$ is nonzero, and therefore $\nabla \boldsymbol{J} \neq 0$. As a result, the current calculated from the current density is not conserved. Therefore, we need to modify the conventional definition of the current density to include the contribution $\rho_{N L}$ of $\rho_{L}$ and induced by the non-local and the local potentials.

We define the new current density in presence of non-local and local potentials as

$$
\boldsymbol{J}_{\text {tot }}=\boldsymbol{J}+\boldsymbol{J}_{N L}+\boldsymbol{J}_{L},
$$

where $\boldsymbol{J}_{L}, \boldsymbol{J}_{N L}$ are the local current density and the non-local current density (We call it the non-local current density because it is merely due to the non-local potential) defined as

$$
\begin{aligned}
& \boldsymbol{J}_{N L}=-\nabla \chi_{N L}(\boldsymbol{r}) \\
& \boldsymbol{J}_{L}=-\nabla \varphi_{L}(\boldsymbol{r}),
\end{aligned}
$$

where $\chi_{N L}, \varphi_{L}$ determined by the following Poisson equation set

$$
\begin{aligned}
& \nabla^{2} \chi_{N L}(\boldsymbol{r})+\rho_{N L}=0 \\
& \nabla^{2} \varphi_{L}(\boldsymbol{r})+\rho_{L}=0,
\end{aligned}
$$

by solving each Poisson equation with proper boundary conditions, we can calculate $\boldsymbol{J}_{N L}$ and $\boldsymbol{J}_{L}$.

It is obvious that the newly defined current density satisfies $\nabla \boldsymbol{J}_{\text {tot }}=0$ and therefore, the calculated current from this current density satisfies the current conservation.

Anywise, in absence of the interactions, the continuity equation takes its simple known form in the quantum mechanics

$$
i \hbar \frac{\partial}{\partial t}|\psi(\boldsymbol{r}, t)|^{2}+\frac{\hbar^{2}}{2 m}\left(\psi^{\dagger}(\boldsymbol{r}, t) \nabla \psi(\boldsymbol{r}, t)-\psi(\boldsymbol{r}, t) \nabla \psi^{\dagger}(\boldsymbol{r}, t)\right)=0 .
$$

\section{Schrödinger Equation in Presence of a Nonlocal Potential in Non-Commutative Phase-Space}

We introduce the non-commutativity in space through the $\star$-product, the Schrödinger equation in presence of non-local and local potentials in noncommutative space is written as

$$
\frac{\boldsymbol{p}^{2}}{2 m} \psi(\boldsymbol{r}, t)+\int V_{N L}\left(\boldsymbol{r}, \boldsymbol{r}^{\prime}\right) \star \psi\left(\boldsymbol{r}^{\prime}, t\right) \mathrm{d} \boldsymbol{r}^{\prime}+V_{L}(\boldsymbol{r}) \star \psi(\boldsymbol{r}, t)=i \hbar \frac{\partial}{\partial t} \psi(\boldsymbol{r}, t),
$$

taking into account that the $\star$-product under the integral sign become ordinary product as shown in Equation (8), leading to found out that the symmetry of the real part of the non-local potential maintained in the non-commutative framework.

For $V_{L}(\boldsymbol{r}) \sim h r$, with $h$ is real-valued, and using Equation (6) we find 


$$
V_{L}(\boldsymbol{r}) \star \psi(\boldsymbol{r}, t)=V_{L}(\boldsymbol{r}) \psi(\boldsymbol{r}, t)+i \Theta_{a b} \partial_{a} V_{L}(\boldsymbol{r}) \partial_{b} \psi(\boldsymbol{r}, t)+0\left(\Theta^{2}\right) .
$$

Then we introduce the non-commutativity in phase by the mapping $\boldsymbol{p} \rightarrow \boldsymbol{p}^{n c}$ through Bopp-shift translation Equation (9), we have

$$
\frac{\left(\boldsymbol{p}^{n c}\right)^{2}}{2 m} \psi(\boldsymbol{r}, t)+\int V_{N L}\left(\boldsymbol{r}, \boldsymbol{r}^{\prime}\right) \star \psi\left(\boldsymbol{r}^{\prime}, t\right) \mathrm{d} \boldsymbol{r}^{\prime}+V_{L}(\boldsymbol{r}) \star \psi(\boldsymbol{r}, t)=i \hbar \frac{\partial}{\partial t} \psi(\boldsymbol{r}, t),
$$

where

$$
\left(\boldsymbol{p}^{n c}\right)^{2}=\left(p_{i}+\frac{1}{2 \hbar} \eta_{i j} r_{j}\right)^{2}=\left(p_{i}\right)^{2}+\frac{1}{2 \hbar} \eta_{i j} p_{i} r_{j}+\frac{1}{2 \hbar} \eta_{i j} p_{j} r_{i}+\frac{1}{4 \hbar^{2}} \eta_{i j} \eta_{i k} r_{j} r_{k},
$$

we restrict ourselves only to the 1st order of the non-commutativity in phase $0\left(\eta^{2}\right)$, [for the equilibrium with the non-commutativity in space considered in this work].

With $\eta_{i j}=-\eta_{j i}=\eta \epsilon_{j i}$, and $\eta_{k}=\frac{1}{2} \epsilon_{k i j} \eta_{i j}$ where $\eta_{i j}=2 \eta_{k} \epsilon_{k i j}$, knowing that $\left(\epsilon_{k i j}\right)^{2}=1$, and $(U \times V)_{k}=\epsilon_{k i j} U_{i} V_{j}, \quad L=\boldsymbol{r} \times \boldsymbol{p}$ then

$$
\begin{aligned}
& \frac{1}{2 \hbar} \eta_{i j} p_{i} r_{j}=\frac{1}{\hbar} \eta_{k} \epsilon_{k i j} p_{i} r_{j}=\frac{1}{\hbar}(\boldsymbol{p} \times \boldsymbol{r}) \boldsymbol{\eta}=-\frac{1}{\hbar} \boldsymbol{L} \boldsymbol{\eta} \\
& \frac{1}{2 \hbar} \eta_{i j} p_{j} r_{i}=-\frac{1}{2 \hbar} \eta_{j i} r_{j} p_{i}=-\frac{1}{\hbar} \eta_{k} \epsilon_{k j i} r_{j} p_{i}=-\frac{1}{\hbar}(\boldsymbol{r} \times \boldsymbol{p}) \boldsymbol{\eta}=-\frac{1}{\hbar} \boldsymbol{L} \boldsymbol{\eta},
\end{aligned}
$$

substituting the above relations in Equation (38). Finally, we obtain

$$
\left(\boldsymbol{p}^{n c}\right)^{2}=\boldsymbol{p}^{2}-\frac{2}{\hbar} \boldsymbol{L} \boldsymbol{\eta}+0\left(\eta^{2}\right) .
$$

Substituting Equations (36)-(40) in Equation (37), we obtain

$$
\begin{aligned}
& -\frac{\hbar^{2}}{2 m} \nabla^{2} \psi(\boldsymbol{r}, t)-\frac{1}{m \hbar} \boldsymbol{L} \boldsymbol{\eta} \psi(\boldsymbol{r}, t)+\int V_{N L}\left(\boldsymbol{r}, \boldsymbol{r}^{\prime}\right) \psi\left(\boldsymbol{r}^{\prime}, t\right) \mathrm{d} \boldsymbol{r}^{\prime}+V_{L}(\boldsymbol{r}) \psi(\boldsymbol{r}, t) \\
& +i \Theta_{a b} \partial_{a} V_{L}(\boldsymbol{r}) \partial_{b} \psi(\boldsymbol{r}, t)=i \hbar \frac{\partial}{\partial t} \psi(\boldsymbol{r}, t) .
\end{aligned}
$$

\subsection{The Frahn-Lemmer Non-local Potential in Non-Commutative Phase-Space}

Knowing that $\int V_{N L}\left(\boldsymbol{r}, \boldsymbol{r}^{\prime}\right) \psi\left(\boldsymbol{r}^{\prime}, t\right) \mathrm{d} \boldsymbol{r}^{\prime}$ goes to $V_{N L}(\boldsymbol{r}, \boldsymbol{p}) \psi(\boldsymbol{r}, t)$, the Schrödinger equation in interaction with Frahn-Lemmer non-local potential and a local potential in non-commutative phase-space is given by

$$
\begin{aligned}
& \frac{\boldsymbol{p}^{2}}{2 m} \psi(\boldsymbol{r}, t)-\frac{1}{m \hbar} \boldsymbol{L} \boldsymbol{\eta} \psi(\boldsymbol{r}, t)+\mathcal{V}_{0} \mathrm{e}^{\left.-\frac{\left(\boldsymbol{p}^{2}-\frac{2}{\hbar} \boldsymbol{L} \eta\right.}{4 \hbar^{2}}\right) \beta^{2}} \psi(\boldsymbol{r}, t)+V_{L}(\boldsymbol{r}) \psi(\boldsymbol{r}, t) \\
& +i \Theta_{a b} \partial_{a} V_{L}(\boldsymbol{r}) \partial_{b} \psi(\boldsymbol{r}, t)=i \hbar \frac{\partial}{\partial t} \psi(\boldsymbol{r}, t),
\end{aligned}
$$

for $\left(\boldsymbol{p}^{2}-\frac{2}{\hbar} \boldsymbol{L} \boldsymbol{\eta}\right) \ll \frac{4 \hbar^{2}}{\beta^{2}}$, let us approximate as

$$
\mathrm{e}^{-\frac{\left(\boldsymbol{p}^{2}-\frac{2}{\hbar} \boldsymbol{L} \eta \beta^{2}\right.}{4 \hbar^{2}}}=1-\frac{\boldsymbol{p}^{2} \beta^{2}}{4 \hbar^{2}}+\frac{\boldsymbol{L} \boldsymbol{\eta} \beta^{2}}{2 \hbar^{3}}
$$


with $a=\frac{\hbar^{2}}{2 m}+\mathcal{V}_{0} \frac{\beta^{2}}{4}, b=\frac{\mathcal{V}_{0} \beta^{2}}{2 \hbar^{3}}-\frac{1}{m \hbar}$ and substituting Equation (43) into Equation (42), we obtain

$$
\begin{aligned}
& -a \nabla^{2} \psi(\boldsymbol{r}, t)+b \boldsymbol{L} \boldsymbol{\eta} \psi(\boldsymbol{r}, t)+\mathcal{V}_{0} \psi(\boldsymbol{r}, t)+V_{L}(\boldsymbol{r}) \psi(\boldsymbol{r}, t) \\
& +i \Theta_{a b} \partial_{a} V_{L}(\boldsymbol{r}) \partial_{b} \psi(\boldsymbol{r}, t)=i \hbar \frac{\partial}{\partial t} \psi(\boldsymbol{r}, t) .
\end{aligned}
$$

The above equation is the non-commutative Schrödinger equation in interaction with Frahn-Lemmer non-local potential and a local potential. The non-commutativity in space influenced the local part, while the noncommutativity in phase touched the non-local part.

\subsection{The Continuity Equation in Non-Commutative Phase-Space}

The Schrödinger equation in presence of non-local and local potentials in non-commutative phase-space is given by the Equation (41), and its complex conjugate is given by

$$
\begin{aligned}
& -\frac{\hbar^{2}}{2 m} \nabla^{2} \psi^{\dagger}(\boldsymbol{r}, t)-\frac{1}{m \hbar} \boldsymbol{L} \boldsymbol{\eta} \psi^{\dagger}(\boldsymbol{r}, t)+\int V_{N L}^{*}\left(\boldsymbol{r}, \boldsymbol{r}^{\prime}\right) \psi^{\dagger}\left(\boldsymbol{r}^{\prime}, t\right) \mathrm{d} \boldsymbol{r}^{\prime} \\
& +V_{L}^{*}(\boldsymbol{r}) \psi^{\dagger}(\boldsymbol{r}, t)-i \Theta_{a b} \partial_{a} V_{L}^{*}(\boldsymbol{r}) \partial_{b} \psi^{\dagger}(\boldsymbol{r}, t)=-i \hbar \frac{\partial}{\partial t} \psi^{\dagger}(\boldsymbol{r}, t),
\end{aligned}
$$

from the multiplications $\psi^{\dagger}(\boldsymbol{r}, t)$ Equation (41) and $\psi(\boldsymbol{r}, t)$ Equation (45), it comes

$$
\begin{aligned}
& -\frac{\hbar^{2}}{2 m} \psi^{\dagger}(\boldsymbol{r}, t) \nabla^{2} \psi(\boldsymbol{r}, t)-\frac{1}{m \hbar} \psi^{\dagger}(\boldsymbol{r}, t) \boldsymbol{L} \boldsymbol{\eta} \psi(\boldsymbol{r}, t) \\
& +\int \psi^{\dagger}(\boldsymbol{r}, t) V_{N L}\left(\boldsymbol{r}, \boldsymbol{r}^{\prime}\right) \psi\left(\boldsymbol{r}^{\prime}, t\right) \mathrm{d} \boldsymbol{r}^{\prime}+\psi^{\dagger}(\boldsymbol{r}, t) V_{L}(\boldsymbol{r}) \psi(\boldsymbol{r}, t) \\
& +i \Theta_{a b} \psi^{\dagger}(\boldsymbol{r}, t) \partial_{a} V_{L}(\boldsymbol{r}) \partial_{b} \psi(\boldsymbol{r}, t)=i \hbar \psi^{\dagger}(\boldsymbol{r}, t) \frac{\partial}{\partial t} \psi(\boldsymbol{r}, t), \\
& -\frac{\hbar^{2}}{2 m} \psi(\boldsymbol{r}, t) \nabla^{2} \psi^{\dagger}(\boldsymbol{r}, t)-\frac{1}{m \hbar} \psi(\boldsymbol{r}, t) \boldsymbol{L} \boldsymbol{\eta} \psi^{\dagger}(\boldsymbol{r}, t) \\
& +\int \psi(\boldsymbol{r}, t) V_{N L}^{*}\left(\boldsymbol{r}, \boldsymbol{r}^{\prime}\right) \psi^{\dagger}\left(\boldsymbol{r}^{\prime}, t\right) \mathrm{d} \boldsymbol{r}^{\prime}+\psi(\boldsymbol{r}, t) V_{L}^{*}(\boldsymbol{r}) \psi^{\dagger}(\boldsymbol{r}, t) \\
& -i \Theta_{a b} \psi(\boldsymbol{r}, t) \partial_{a} V_{L}^{*}(\boldsymbol{r}) \partial_{b} \psi^{\dagger}(\boldsymbol{r}, t)=-i \hbar \psi(\boldsymbol{r}, t) \frac{\partial}{\partial t} \psi^{\dagger}(\boldsymbol{r}, t),
\end{aligned}
$$

then, by the subtraction of Equation (46) from Equation (47) we obtain

$$
\begin{aligned}
& -\frac{\hbar^{2}}{2 m} \nabla\left(\psi^{\dagger}(\boldsymbol{r}, t) \nabla \psi(\boldsymbol{r}, t)-\psi(\boldsymbol{r}, t) \nabla \psi^{\dagger}(\boldsymbol{r}, t)\right) \\
& +\frac{1}{m \hbar}\left(\psi(\boldsymbol{r}, t) \boldsymbol{L} \boldsymbol{\eta} \psi^{\dagger}(\boldsymbol{r}, t)-\psi^{\dagger}(\boldsymbol{r}, t) \boldsymbol{L} \boldsymbol{\eta} \psi(\boldsymbol{r}, t)\right) \\
& +\int\left(\psi^{\dagger}(\boldsymbol{r}, t) V_{N L}\left(\boldsymbol{r}, \boldsymbol{r}^{\prime}\right) \psi\left(\boldsymbol{r}^{\prime}, t\right)-\psi(\boldsymbol{r}, t) V_{N L}^{*}\left(\boldsymbol{r}, \boldsymbol{r}^{\prime}\right) \psi^{\dagger}\left(\boldsymbol{r}^{\prime}, t\right)\right) \mathrm{d} \boldsymbol{r}^{\prime} \\
& +\psi^{\dagger}(\boldsymbol{r}, t) V_{L}(\boldsymbol{r}) \psi(\boldsymbol{r}, t)-\psi(\boldsymbol{r}, t) V_{L}^{*}(\boldsymbol{r}) \psi^{\dagger}(\boldsymbol{r}, t) \\
& +i \Theta_{a b}\left(\psi^{\dagger}(\boldsymbol{r}, t) \partial_{a} V_{L}(\boldsymbol{r}) \partial_{b} \psi(\boldsymbol{r}, t)+\psi(\boldsymbol{r}, t) \partial_{a} V_{L}^{*}(\boldsymbol{r}) \partial_{b} \psi^{\dagger}(\boldsymbol{r}, t)\right) \\
& =i \hbar \frac{\partial}{\partial t}\left(\psi^{\dagger}(\boldsymbol{r}, t) \psi(\boldsymbol{r}, t)\right),
\end{aligned}
$$


contracting the above equation as follows

$$
\frac{\partial \rho}{\partial t}+\nabla \boldsymbol{J}+\rho_{N L}+\rho_{L}^{n c}+\mathcal{C}^{n c}=0,
$$

Equation (48) will be recognized as the non-commutative continuity equation, denoting the separate terms in it as follows

$$
\begin{aligned}
& \rho=J^{0}=\psi^{\dagger}(\boldsymbol{r}, t) \psi(\boldsymbol{r}, t)=|\psi(\boldsymbol{r}, t)|^{2} \\
& \boldsymbol{J}=\frac{-\hbar^{2}}{2 m}\left(\psi^{\dagger}(\boldsymbol{r}, t) \nabla \psi(\boldsymbol{r}, t)-\psi(\boldsymbol{r}, t) \nabla \psi^{\dagger}(\boldsymbol{r}, t)\right) \\
& \rho_{N L}=\int\left(\psi^{\dagger}(\boldsymbol{r}, t) V_{N L}\left(\boldsymbol{r}, \boldsymbol{r}^{\prime}\right) \psi\left(\boldsymbol{r}^{\prime}, t\right)-\psi(\boldsymbol{r}, t) V_{N L}^{*}\left(\boldsymbol{r}, \boldsymbol{r}^{\prime}\right) \psi^{\dagger}\left(\boldsymbol{r}^{\prime}, t\right)\right) \\
& \rho_{L}=\psi^{\dagger}(\boldsymbol{r}, t) V_{L}(\boldsymbol{r}) \psi(\boldsymbol{r}, t)-\psi(\boldsymbol{r}, t) V_{L}^{*}(\boldsymbol{r}) \psi^{\dagger}(\boldsymbol{r}, t) \\
& \rho_{L}^{\Theta}=i \Theta_{a b}\left(\psi^{\dagger}(\boldsymbol{r}, t) \partial_{a} V_{L}(\boldsymbol{r}) \partial_{b} \psi(\boldsymbol{r}, t)+\psi(\boldsymbol{r}, t) \partial_{a} V_{L}^{*}(\boldsymbol{r}) \partial_{b} \psi^{\dagger}(\boldsymbol{r}, t)\right) \\
& \mathcal{C}^{n c}=\frac{1}{m \hbar}\left(\psi(\boldsymbol{r}, t) \boldsymbol{L} \boldsymbol{\eta} \psi^{\dagger}(\boldsymbol{r}, t)-\psi^{\dagger}(\boldsymbol{r}, t) \boldsymbol{L} \boldsymbol{\eta} \psi(\boldsymbol{r}, t)\right) \\
& \rho_{L}^{n c}=\rho_{L}+\rho_{L}^{\Theta} .
\end{aligned}
$$

It is obvious that the conservation of the current density in the non-commutative phase-space completely violated, which means that the current density does not satisfy the current conservation. Then we move to the interpretation of the separating terms, the existence of the quantities corresponding to the explicit $\Theta$, $\eta$ parameters, which are involved in the obtained Equation (50) due to the effect of the phase-space non-commutativity on the Schrödinger equation. Firstly, these quantities emerged merely as terms containing the parameters $\Theta, \eta$ consequently after extracting the non-commutative continuity equation, those terms being responsible for generating the new quantities collectively with the correction term that contains $\Theta$ parameter.

More accurately, the effect of the non-local potential on the continuity equation arises as a non-local quantity of density type, as well as for the locality effect, it appears as a local quantity of density type also, where the non-commutativity in phase formed only a correction term $\mathcal{C}^{n c}$, which appeared in the non-commutative continuity equation, but for the non-commutativity in space affected only the local quantity $\rho_{L}^{n c}$ through a first-order correction. Once the local potential is null, the local density quantity with its non-commutative correction will disappear.

Comparing the continuity equation in commutative and in non-commutative cases, we find that the non-commutativity influence is very clear in the amount of the local potential, but for the non-locality amount vanishes, we find that the non-commutativity effect violates the conservation of the continuity equation.

In what follows, we modify the expression of the density current, in which, it will be conserved in the non-commutative phase-space:

If the local potential $V_{L}(\boldsymbol{r})$ is real, the quantity $\rho_{L}^{n c}$ vanishes (similar to the commutative case), also when the non-local potential $V_{N L}\left(\boldsymbol{r}, \boldsymbol{r}^{\prime}\right)$ is a real diagonal matrix, the quantity $\rho_{N L}$ vanishes. But the current calculated from $\boldsymbol{J}$ is not conserved due to the phase non-commutativity correction $\mathcal{C}^{\text {nc }}$. However, 
while $\rho_{N L}$ and $\rho_{L}$ are nonzero. As a result, the current density not conservable, as well as of the symmetry is not maintained in the non-commutative phase-space. Therefore, we need to modify the conventional definition of the current density to include the contribution of $\rho_{N L}$ and $\rho_{L}^{n c}$, and in the steady state $\frac{\partial \rho}{\partial t}=0$, Equation (49) becomes

$$
\nabla J+\rho_{N L}+\rho_{L}^{n c}+C^{n c}=0 .
$$

We make the following replacement

$$
\boldsymbol{J}+\mathcal{K}^{n c}=\mathcal{J}^{n c} \text {, with } C^{n c}=\mathcal{J K} \mathcal{K}^{n c},
$$

with the condition $\nabla C^{n c}=0$. We define the new global current density in presence of a non-local and a local potential where the non-commutativity is considered, as

$$
\boldsymbol{J}_{\text {tot }}^{n c}=\mathcal{J}^{n c}+\boldsymbol{J}_{N L}+\boldsymbol{J}_{L}^{n c} \text {, }
$$

where $\boldsymbol{J}_{L}^{n c}, \boldsymbol{J}_{N L}$ are the non-commutative local current density, and the non-local current density defined as

$$
\begin{aligned}
& \boldsymbol{J}_{N L}=-\nabla \chi_{N L}(\boldsymbol{r}) \\
& \boldsymbol{J}_{L}^{n c}=-\nabla \varphi_{L}^{n c}(\boldsymbol{r})^{\prime}
\end{aligned}
$$

where $\chi_{N L}, \varphi_{L}^{n c}$ determined by the following Poisson equation set

$$
\begin{aligned}
& \nabla^{2} \chi_{N L}(\boldsymbol{r})+\rho_{N L}=0 \\
& \nabla^{2} \varphi_{L}^{n c}(\boldsymbol{r})+\rho_{L}^{n c}=0^{\prime}
\end{aligned}
$$

by solving each Poisson equation through the proper boundary conditions, we obtain $\boldsymbol{J}_{N L}$ and $\boldsymbol{J}_{L}^{n c}$. Therefore, the newly defined total current density satisfies $\nabla \boldsymbol{J}_{\text {tot }}^{n c}=0$ and therefore, the calculated non-commutative current from this current density satisfies the current conservation.

\section{Conclusions}

In conclusion, the phase-space non-commutativity introduced in the Schrödinger equation and consequently, the continuity equation obtained in the case of commutativity and in the case of non-commutativity, without forgetting that the Schrödinger equation considered in interaction with non-local and local potentials, this, in turn, is responsible for causing new quantities of density type in the continuity equation. We found that the non-commutativity in phase-space is not suitable for describing the current density in presence of non-local and local potentials. Knowing that the phase-space non-commutativity effect introduced through both of the Bopp-shift linear translation method and the Moyal-Weyl product, under the condition that space-space and momentum-momentum are all commutative, the results in non-commutative phase-space return to that of the usual quantum mechanics.

The results of the present work can be used to investigate the conservation laws by involving the non-commutative geometry such as the non-commutative 
CPT symmetry (with the Lorentz invariance), the conservation of weak isospin (with $S U(2)$, Gauge invariance). In the electromagnetism also such as the non-commutative Maxwell's equations, maybe also used in the non-commutative general relativity. We are out looking to investigate the Klein-paradox depending on these results.

\section{Acknowledgements}

The author would like to thank Jane Gao Managing Editor of JMP for his help and support.

\section{Conflicts of Interest}

The authors declare no conflicts of interest regarding the publication of this paper.

\section{References}

[1] Galperin, M., Nitzan, A. and Ratner, M.A. (2007) Heat Conduction in Molecular Transport Junctions. Physical Review B, 75, Article ID: 155312. https://doi.org/10.1103/PhysRevB.75.155312

[2] Nayak, G.C. (2013) Holographic Aspects of a Relativistic Nonconformal Theory. Advances in High Energy Physics, 2013, Article ID: 389541. https://doi.org/10.1155/2013/389541

[3] Nayak, G.C. (2013) General Form of Color Charge of the Quark. European Physical Journal C, 73, 2442. https://doi.org/10.1140/epjc/s10052-013-2442-6

[4] Coz, M., Arnold, L.G. and MacKellar, A.D. (1970) Nonlocal Potentials and Their Local Equivalents. Annals of Physics, 59, 219-247. https://doi.org/10.1016/0003-4916(70)90401-X

[5] Pattanayak, D.N. and Wolf, E. (1976) Scattering States and Bound States as Solutions of the Schrödinger Equation with Nonlocal Boundary Conditions. Physical Review D, 13, 913-923. https://doi.org/10.1103/PhysRevD.13.913

[6] Kidun, O., Fominykh, N. and Berakdar, J. (2002) Scattering and Bound-State Problems with Non-Local Potentials: Application of the Variable-Phase Approach. Journal of Physics A: Mathematical and General, 35, 9413. https://doi.org/10.1088/0305-4470/35/44/310

[7] Reeves, M. and Owen, L.W. (1969) Solution of a Schroedinger Equation with a Nonlocal Potential. Journal of Computational Physics, 4, 572-578. https://doi.org/10.1016/0021-9991(69)90021-7

[8] Taylor, J., Guo, H. and Wang, J. (2001) Ab Initio Modeling of Quantum Transport Properties of Molecular Electronic Devices. Physical Review B, 63, Article ID: 245407. https://doi.org/10.1103/PhysRevB.63.245407

[9] Balantekin, A.B., Beacom, J.F. and Cândido Ribeiro, M.A. (1998) Green's Function for Nonlocal Potentials. Journal of Physics G: Nuclear and Particle Physics, 24, 2087. https://doi.org/10.1088/0954-3899/24/11/010

[10] Perey, F. and Buck, B. (1962) A Non-Local Potential Model for the Scattering of Neutrons by Nuclei. Nuclear Physics, 32, 353-380. https://doi.org/10.1016/0029-5582(62)90345-0

[11] Fetter, A.L. and Walecka, J.D. (1971) Quantum Theory of Many-Particle Systems. 
McGraw-Hill, New York.

[12] Feshbach, H. (1958) Unified Theory of Nuclear Reactions. Annals of Physics, 5, 357-390. https://doi.org/10.1016/0003-4916(58)90007-1

[13] Satchler, G.R. (1983) Direct Nuclear Reactions. Clarendon, Oxford.

[14] Connes, A. (2000) A Short Survey of Noncommutative Geometry. Journal of Mathematical Physics, 41, 3832. https://doi.org/10.1063/1.533329

[15] Chamseddine, A.H., Connes, A. and Mukhanov, V. (2015) Quanta of Geometry: Noncommutative Aspects. Physical Review Letters, 114, Article ID: 091302. https://doi.org/10.1103/PhysRevLett.114.091302

[16] Kastler, D. (2000) Noncommutative Geometry and Fundamental Physical Interactions: The Lagrangian Level-Historical Sketch and Description of the Present Situation. Journal of Mathematical Physics, 41, 3867. https://doi.org/10.1063/1.533330

[17] Madore, J. (1998) Introduction to Non-Commutative Geometry. Proceedings of Science Corfu 98, 16, 1-19.

[18] Seiberg, N. and Witten, E. (1999) String Theory and Noncommutative Geometry. Journal of High Energy Physics, 1999, Article No. 32. https://doi.org/10.1088/1126-6708/1999/09/032

[19] Bastos, C. and Bertolami, O. (2008) Berry Phase in the Gravitational Quantum Well and the Seiberg-Witten Map. Physics Letters A, 372, 5556-5559. https://doi.org/10.1016/j.physleta.2008.06.073

[20] Bertolami, O., Rosa, J.G., de Aragão, C.M.L., Castorina, P. and Zappalà, D. (2005) Noncommutative Gravitational Quantum Well. Physical Review D, 72, Article ID: 025010. https://doi.org/10.1103/PhysRevD.72.025010

[21] Yang, Z.H., Long, C.Y., Qin, S.J. and Long, Z.-W. (2010) DKP Oscillator with Spin-0 in Three-Dimensional Noncommutative Phase Space. International Journal of Theoretical Physics, 49, 644-651. https://doi.org/10.1007/s10773-010-0244-2

[22] Haouam, I. and Chetouani, L. (2018) The Foldy-Wouthuysen Transformation of the Dirac Equation in Noncommutative Phase-Space. Journal of Modern Physics, 9 , 2021-2034. https://doi.org/10.4236/jmp.2018.911127

[23] Haouam, I. (2019) The Non-Relativistic Limit of the DKP Equation in Non-Commutative Phase-Space. Symmetry, 11, 223. https://doi.org/10.3390/sym11020223

[24] Haouam, I. (2018) The Phase-Space Noncommutativity Effect on the Large and Small Wave-Function Components Approach at Dirac Equation. Open Access Library Journal, 5, e4108.

[25] Curtright, T., Fairlie, D. and Zachos, C. (1998) Features of Time-Independent Wigner Functions. Physical Review D, 58, Article ID: 025002. https://doi.org/10.1103/PhysRevD.58.025002

[26] Horiuchi, H. (1980) A Semi-Classical Treatment of Non-Local Potentials. Progress of Theoretical Physics, 64, 184-203. https://doi.org/10.1143/PTP.64.184

[27] Frahn, W.E. and Lemmer, R.H. (1957) Velocity-Dependent Nuclear Interaction. II Nuovo Cimento, 6, 1221-1224. https://doi.org/10.1007/BF02747404

[28] Li, C.S., Wan, L.H., Wei, Y.D. and Wang, J. (2008) Definition of Current Density in the Presence of a Non-Local Potential. Nanotechnology, 19, Article ID: 155401. https://doi.org/10.1088/0957-4484/19/15/155401 